\title{
Memory-Based Local Search for Simplified Protein Structure Prediction
}

\author{
Swakkhar Shatabda ${ }^{*}$, M.A.Hakim Newton, Duc Nghia Pham and Abdul Sattar \\ Queensland Research Laboratory, NICTA \\ Institute for Integrated and Intelligent Systems, Griffith University
}

\begin{abstract}
Protein structure prediction is one of the most challenging problems in computational biology. Given a protein's amino acid sequence, a simplified version of the problem is to find an on-lattice self-avoiding walk that minimizes the interaction energy among the amino acids. In this paper, we present a memory-based local search method for the simplified problem using Hydrophobic-Polar energy model and Face Centered Cubic lattice. By memorizing local minima and then avoiding their neighbohood, our approach significantly improves the state-of-the-art local search method for protein structure prediction on a set of standard benchmark proteins.
\end{abstract}

\section{Categories and Subject Descriptors}

I.2 [Computing Methodologies]: Artificial Intelligence; [Problem Solving, Control Methods, and Search]: Heuristic methods;

J.3 [Computer Applications]: Life and Medical Sciences; [Biology and Genetics]: protein structure prediction

\section{General Terms}

Algorithms

\section{Keywords}

Local Search, Memory-Based Search, HP Energy Model, FCC Lattice, Protein Structure Prediction

\footnotetext{
* corresponding author

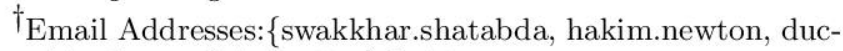
nghia.pham, abdul.sattar\}@nicta.com.au

$\ddagger_{\text {NICTA is funded by the Australian Government as repre- }}$ sented by the Department of Broadband, Communications and the Digital Economy and the Australian Research Council through the ICT Centre of Excellence program.
}

\section{INTRODUCTION}

Local search methods for the protein structure prediction (PSP) problem have shown promising results in recent years $[25,14,15,36]$. Given a protein's amino acid sequence, a simplified version of the PSP problem is to find an on-lattice self-avoiding walk that minimizes the interaction energy among the amino acid monomers. Local search methods are very quick in finding reasonably good solutions. However, they in general suffer from re-visitation and stagnation. When applied to large proteins, they often struggle to escape from local minima although they find near optimal energy values for small proteins (with less than 200 monomers).

To handle these difficulties in PSP, researchers $[14,15]$ have used tabu lists, adaptive or reactive measures, and various restarting mechanisms. Similar approaches have also been used in other domains such as propositional satisfiability [28] and quadratic assignment problem [6]. Moreover, there are several other approaches that make use of a cache of nogoods (e.g. conflict clauses in satisfiability, violating constraints and culprit variables in constraint problems) [17, 20]. However, these nogoods are used in capturing infeasibility and pruning the search space thereof, and thus bringing somewhat systematic nature within the search.

In this paper, we present a memory-based method to enhance the performance of local search algorithms for the simplified PSP problem. During the search, our method memorizes a proportion of the local minima visited and avoids future re-visitation of the proximate region of these stored local minima. Storing local minima is similar to caching of nogoods, but discarding the conformations of this large region risks obtaining the global minimum. The local minima are represented by the whole solutions. Using partial solutions seems to be not useful as the nature of the problem largely depends on the placements of all amino acids in the sequence and their interactions. The novelty of our method mainly lies in the purpose and usage of the local minima stored during the search for PSP in particular and other search problems in general.

We implemented our method on top of the state-of-the-art local search algorithm [14] for the simplified PSP problem using Hydrophobic-Polar (HP) energy model on the Face Centered Cubic (FCC) Lattice. We represent protein conformations (i.e. the structures produced by the on-lattice self-avoiding walk) using a relative encoding (based on relative directions between the positions on the lattice of consecutive amino acids). We use hamming distance to check the similarity between two conformations and discard any 
conformation that is found within the proximity of a stored local minimum. Our experimental results show that our method achieves a significant improvement over state-of-theart solvers on standard benchmark proteins.

The rest of the paper is organized as follows: Section 2 describes the PSP problem; Section 3 reviews the related work; Section 4 describes the local search framework; Section 5 describes our approach in detail; Section 6 discusses and analyzes the experimental results; and finally, Section 7 presents our conclusions and outlines our future work.

\section{PROTEIN STRUCTURE PREDICTION}

Proteins are the fundamental component of all living cells, participating virtually in every process within the cell including carrying oxygen (by hemoglobin), signaling cells (by insulin), fighting infection (by antibodies) and performing metabolism (by enzymes). Proteins are sequences of amino acids (or monomers). Since Anfinsen's famous thermodynamic hypothesis [2], it has been believed that every protein has a unique and stable native three dimensional (3D) structure. The native structure has the lowest free energy possible. A protein needs to fold into its native structure to function properly. Mis-folded proteins cause many critical diseases such as Alzheimer's disease, Cystic fibrosis, and Mad Cow disease.

Proper knowledge of the native structure of a protein is of paramount importance in understanding the very basics of life and can have an enormous impact in the field of drug discovery of the related diseases. Due to the complex and dynamic nature of the folding process, and the unknown contributing factors of the energy function, PSP is one of the hardest problems in biology. Even the in vitro laboratory methods like X-ray crystallography and Nuclear Magnetic Resonance (NMR) spectroscopy are much slow and expensive [5]. These issues have attracted researchers from other fields $[24,21,22,16]$ to approach the problem using their own techniques.

A significant number of computational methods have been applied in PSP. Computational methods like homology modeling [5] and protein threading [32] depend on the templates (or structures) of known proteins while ab initio methods start from the basic principles and rely only on the given amino acid sequence. The PSP problem can be viewed as a search problem, where one has to find a stable, unique and kinetically accessible native structure from the space of all possible structures (also called conformations) regardless of how detailed the given model is. Unfortunately even for the simplified models, systematic search techniques are almost impractical since they perform exhaustive search and requires a huge amount of computational resources. In contrast, local search methods are normally very quick in finding a solution, although they suffer from re-visitation and stagnation, and require good heuristics.

Nevertheless, the performance of computational methods often degrades when applied to predict the real structures of proteins. There are two reasons behind this: the contributions of different forces to the free energy that stabilize the folded conformation are poorly understood, and the space of possible conformations is very large and complex. Moreover, very detailed atomic level models of proteins require huge amount of computational effort. Consequently, these have led researchers to model the problem in a simplified way e.g. by using lattice-based structures and low resolu- tion energy functions. The simplified models, though lack many details, produce a realistic backbone for a protein. A backbone structure can be further refined to include more details by hierarchical methods [31].

\subsection{Simplified Model}

In a simplified model, all monomers have an equal size and all bonds are of an equal length. Each amino acid monomer is represented by a single point and its position is restricted to a three dimensional lattice. A simplified energy function is used in calculating the energy of a conformation. The given amino acid sequence fits into a fixed lattice, where every two consecutive monomers in the sequence are neighbors to each other on the lattice (called the chain constraint) and two monomers can not occupy the same lattice point (called the self avoiding constraint).

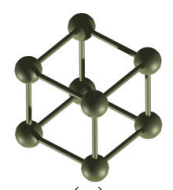

(a)

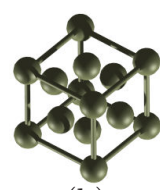

(b)
Figure 1: Different 3D lattices: (a) cubic lattice and (b) face centered cubic lattice

Lattice. A lattice $\mathbb{L}$ is a discretization of the continuum of real space where all lattice points are generated by some basis vectors $\vec{v}_{1}, \cdots \vec{v}_{k}$. Two lattice points $p, q \in \mathbb{L}$ are said to be in contact or neighbors of each other, if $q=p+\overrightarrow{v_{i}}$ for some vector $\vec{v}_{i}$ in the basis of $\mathbb{L}$. Protein conformations are represented by a sequence of basis vectors (absolute encodings [37]), or by the relative vectors that are relative to the previous basis vectors in the sequence (relative encodings [4]). The Face Centered Cubic (FCC) lattice is preferred to cubic lattice (see Figure 1) due to the parity problem [13]. Moreover, the FCC lattice provides the densest packing [11] for spheres of equal size, and the highest degree of freedom for placing an amino acid monomer. Thus, it can provide a realistic discrete mapping for proteins. The FCC lattice is generated by the following basis vectors: $\overrightarrow{v_{1}}=(1,1,0)$, $\overrightarrow{v_{2}}=(-1,-1,0), \overrightarrow{v_{3}}=(-1,1,0), \overrightarrow{v_{4}}=(1,1,0), \overrightarrow{v_{5}}=(0,1,1)$, $\overrightarrow{v_{6}}=(0,1,-1), \overrightarrow{v_{7}}=(1,1,0), \overrightarrow{v_{8}}=(1,0,-1), \overrightarrow{v_{9}}=(0,-1,1)$, $\overrightarrow{v_{10}}=(-1,0,1), \overrightarrow{v_{11}}=(0,-1,-1), \overrightarrow{v_{12}}=(-1,0,-1)$. The co-ordination number of the FCC lattice is 12 ; which means a point in the lattice has twelve neighbors.

Energy Model. Lau and Dill [24] proposed a simplified Hydrophobic-Polar (HP) energy model. In the HP model, all the amino acids are divided into two groups: hydrophobic $(\mathrm{H})$, and hydrophilic or polar $(\mathrm{P})$. The amino acid sequence of a protein is represented as a string $s$ of the alphabet $\{H, P\}$. The free energy calculation for the HP model, shown in (1), counts only the energy interactions between two non-consecutive amino acid monomers.

$$
E=\sum_{i, j: i+1<j} c_{i j} \cdot e_{i j}
$$

Here, $c_{i j}=1$ only if two monomers $i$ and $j$ are neighbors (or in contact) on the lattice or 0 otherwise. The other term, $e_{i j}$ is calculated depending on the type of amino acids: $e_{i j}=-1$ if $s_{i}=s_{j}=H$ or 0 otherwise. Minimizing the 
summation in (1) is equivalent to maximizing the number of non-consecutive $\mathrm{H}-\mathrm{H}$ contacts. There are other simplified energy models in the literature, e.g. variants of HP-model [9], and MJ matrix model [29].

Using the HP energy model together with the FCC lattice, the simplified PSP problem is defined as: given a sequence $s$ of length $n$, find a self avoiding walk $p_{1} \cdots p_{n}$ on the lattice that minimizes the energy defined by (1).

\section{RELATED WORK}

The protein structure prediction problem is a hard combinatorial problem, even for the simple square [12] and cubic lattices [7]. Yue and Dill [40] first introduced the ConstraintBased Hydrophobic Core Construction (CHCC) method to find the optimal solution in cubic lattice and HP energy model. The CHCC method supported only HP model and could not report degeneracy or non-unique structures for several protein sequences. The Constraint-based Protein Structure Prediction (CPSP) tool introduced by Backofen and Will [27] provides a solution to these problems. Palu et al. [30] used biologically inspired constraints and highly optimized propagators to obtain satisfactory results on small and medium sized instances. They developed a constraint solver [1] named COLA, that produced acceptable quality solutions for relatively larger protein sequences (length < $80)$.

Lesh et al. [25] used tabu search on a novel set of lattice transformation called pull moves for two-dimensional HP model with square lattice. Blazewicz et al. [8] also implemented tabu search meta-heuristics independently of that of Lesh et al. [25]. Unger and Moult [37] were first to apply genetic algorithms to solve the problem. Later, Hoque et al. [18] improved genetic algorithms by using hydrophobic core-directed moves and twin removal techniques. Jiang et al. [19] combined tabu search strategy with genetic algorithms in the two-dimensional HP Model. Most of these algorithms were tested on small proteins $($ length $=48)$ from the famous Harvard benchmark [41].

Hybrid techniques combine the power of different strategies to gain better results. Using the pull moves, Klau et al. [23] proposed an interactive optimization framework called Human Guided Simple Search (HuGS). Using the same pull move set, Ullah et al. [35] proposed a two-stage optimization approach. It used the CPSP tool [27] to provide the initial structure for local search procedure on FCC lattice. Furthermore, Ullah et al. [36] combined local search and constraint programming approaches. They introduced a protein folding simulation procedure on FCC lattice and employed the COLA solver [1] to generate neighborhood states for a simulated annealing based local search. The MJ matrix comprising pairwise interactions between 20 amino acids was used. They tested their approaches on some real proteins $($ length < 80) from the Protein Data Bank (PDB).

Cebrian et al. [10] used tabu search to find 3D structures of Harvard instances [41] on FCC lattices for the first time. In their subsequent work, Dotu et al. [14, 15] applied Large Neighborhood Search (LNS) to further optimize the results found in [10]. They also improved the tabu search by adopting a new neghborhood selection technique [15]. Both of their works are implemented in COMET [38]. Other methods (such as Simulated Annealing [21], Ant Colony Optimization (ACO) [33], and Extremal Optimization [26]) are also found in the literature.

\section{LOCAL SEARCH FRAMEWORK}

In this section, we describe the local search framework proposed by Dotu et al. [15]. The algorithm depends on a randomized structured initialization function, a new fitness function that is different from the HP energy model, and a specialized neighborhood switching policy for move selection with a tabu list. In this framework, only moves involving single monomer are allowed. For any given conformation $c$ and a sequence position $i$, a move $(i, p, c)$ that moves an amino acid $i$ to a new position $p$ is allowed if $p$ is free and is in contact with both amino acids at positions $i-1$ and $i+1$, and $i$ is not in the tabu list. The length of the tabu list takes a random value from $[4, n / 4]$, where $n$ is the length of the sequence. A move is called a $\mathrm{H}$-move or $\mathrm{P}$-move depending on the type of the monomer to be moved. The fitness function minimizes the summation of $\mathrm{HH}$-distances for all non-consecutive pairs of $\mathrm{H}$-monomers and thus guides the search. The quality of the solutions is still determined by the HP energy value. The fitness function can be formally defined as the following:

$$
f(c)=\sum_{i, j: i+1<j}^{n}(d v(i, j))^{2} \times\left(s_{i}=H, s_{j}=H\right)
$$

where $d v(i, j)=d(i, j)^{2}-2$ and $d(i, j)=\left(x_{i}-x_{j}\right)^{2}+$ $\left(y_{i}-y_{j}\right)^{2}+\left(z_{i}-z_{j}\right)^{2}$, i.e. square of the Euclidean distance between the $i$ th and $j$ th amino acids in the current conformation $c$ of a sequence $s$ of length $n$. The algorithm is similar to that depicted in Algorithm 1 except the selectMove() method at line 8 and at lines 11-13 where a local minimum is stored. The counter nonImp counts the non-improving steps after the latest best solution is found and stagnation is detected using the parameter maxStable. Every time the search faces stagnation, it restarts from the previously found best solution. The neighborhood selection policy periodically switches between two kinds of moves available (see Algorithm 2 for exact details). While selecting from the available $\mathrm{P}$-moves, it selects randomly and in the case of $\mathrm{H}$ moves it selects the move that minimizes the fitness function. By the virtue of this fitness function, the algorithm quickly pulls H-monomers closer to form a compact core and thus achieves better solutions compared to when used with only the basic HP energy model. Currently, this approach [15] produces the best results for the benchmark proteins used with HP model and FCC lattice.

\section{OUR MEMORY-BASED APPROACH}

Local search algorithms in general suffer from stagnation and re-visitation, and perform poorly on large size proteins. To tackle the drawbacks, these algorithms use tabu lists and restart mechanisms. Moreover, a certain level of revisitation is allowed during the search. The rationale behind this is: if the neighborhood generation and/or their evaluation is not computationally expensive then number of re-visitation will not hamper the performance of the solver. Moreover, at each restart the search is assumed to start from a new region in the search space.

The search framework in [15] maintains a tabu list as memory to prevent re-visitation. Adaptive or reactive tabu search algorithms have been applied in other domains [6], where they also keep a small list of recently visited solutions to dynamically adjust the tabu size. Such techniques can 

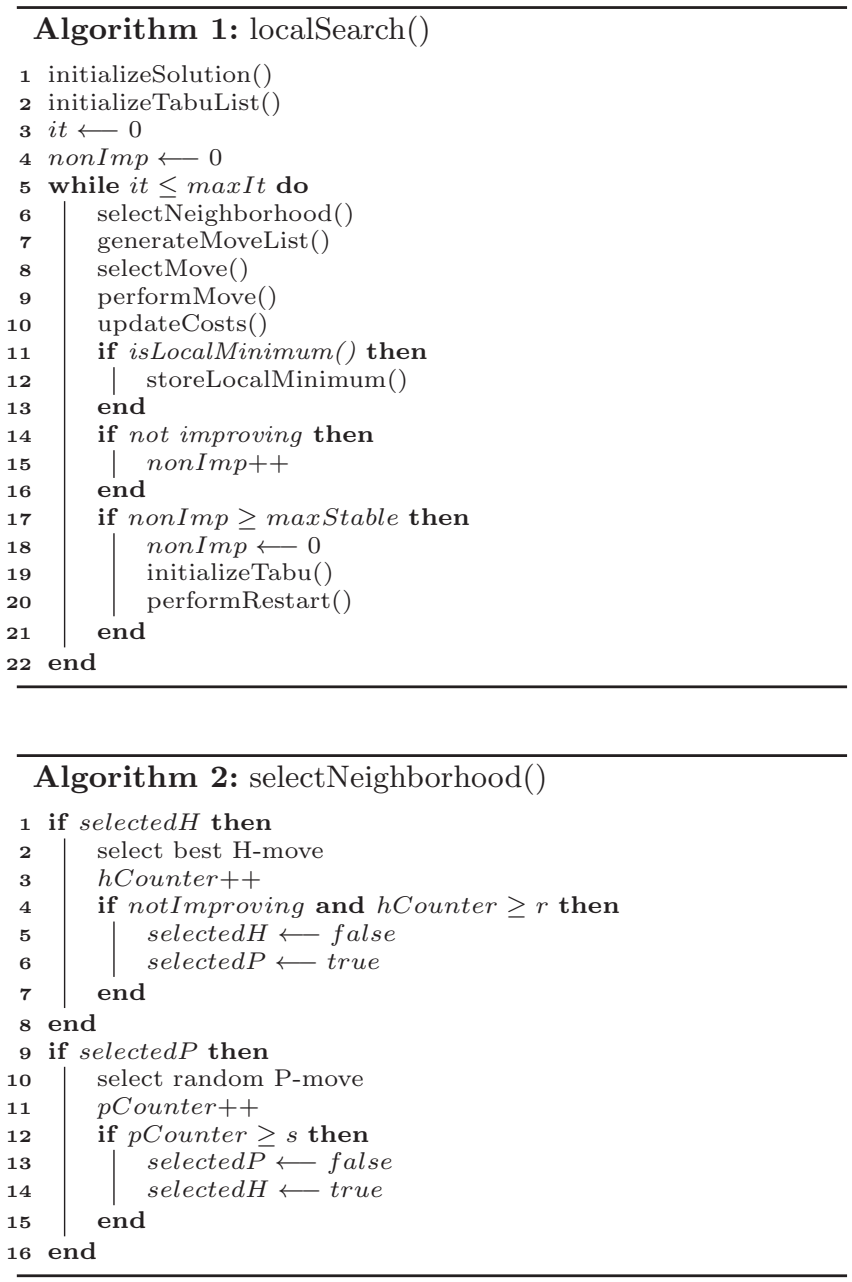

eliminate only those re-visitations that occur within a short period of time; long term re-visitations still occur. Even at the time of the restarts or while following alternative paths, the search visits the same region in the search space again and again; which is not prevented effectively. Few other approaches are found in the literature that maintain a cache of nogood solutions to prune the search space [17, 20]. Most of these methods are systematic in nature and maintain a cache of partial solutions which are called nogoods.

The fitness function used in [15] has an interesting property to note. Since it minimizes the distance between two H-monomers, it quickly forms a hydrophobic core. It is observed that the fitness function guides the H-type amino acids to be placed closer to each other and form a compact core at the center of the structure. Once the core is formed, it takes a significant amount of perturbations to destroy that compact core and find an alternative core to achieve a better solution. Moreover, there are cases where multiple cores are formed and they have to be broken in order to merge. The nature of the fitness function (depicted in Figure 2) suggests that the search oscillates within the region it is currently in, and improves if it can eventually escape that area. Even for a large tabu tenure the number of re-visitations is significantly large compared to the number of generated solutions. In Figure 2, we report re-visitations of the tabu search [15] for four different protein or protein-like sequences. The first

\begin{tabular}{|c|c|c|c|}
\hline \multicolumn{2}{|c|}{ Protein } & \multirow{2}{*}{\begin{tabular}{|c|} 
Tabu \\
Len
\end{tabular}} & \multirow{2}{*}{$\begin{array}{l}\text { Dupl- } \\
\text { icates }\end{array}$} \\
\hline Seq & Len & & \\
\hline $\mathrm{H} 1$ & 48 & 9 & $40.92 \%$ \\
\hline P3 & 81 & 17 & $31.19 \%$ \\
\hline S1 & 135 & 25 & $42.53 \%$ \\
\hline $\mathrm{R} 1$ & 200 & 28 & $39.34 \%$ \\
\hline
\end{tabular}

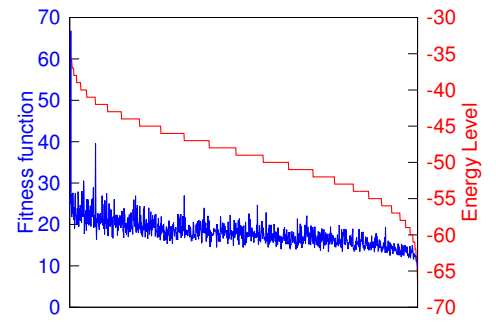

Figure 2: i) Proportion of duplicated conformations in the first one million conformations generated by tabu search [15] (left), ii) the plot of energy level and fitness function over 100,000 iterations of the tabu search algorithm [15] for protein sequence H1 (length $=48$ ) sequence sorted by energy level; fitness function scaled down by 1000 (right).

sequence with length 48 is taken from the famous tortilla benchmarks [41], the other three sequences are taken from Sebastian Will's PhD thesis [39].

This huge number of re-visitations pose several problems regarding the performance of the local search. The algorithm spends a significant amount of time to generate duplicate solutions, which if prevented, can lead the search to new regions in the search space. The fitness function calculation for the generated conformations is also very often computationally expensive even for several simplified models. We

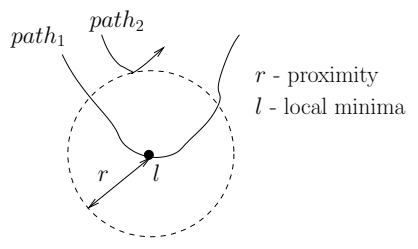

Figure 3: Nature of the memory-based search.

suggest that by memorizing representative local minima of a specific region of the search space, it is possible to eliminate the re-visitation of its proximate search region. For this particular algorithm, since there is only a single monomer change in two successive conformations, we are able to eliminate a large neighborhood of the local minima depending on the similarity measure and the number of local minima stored. In each iteration, while selecting the candidates or after generating them, these candidates are discarded if they are found to be within a pre-defined proximity of any of the stored local minima and the search is diverted (see Figure $3)$. If any search path path $_{2}$ falls within the proximity measure $r$ of a local minimum $l$, found by a previous search path path $_{1}$, the search is diverted from the proximate region of that local minimum. The similarity measure depends on the representation of the protein conformation.

We integrate our technique with Dotu et al.'s algorithm [15]. The differences are depicted in Algorithm 1: how to detect a local minimum and decide to store it (lines 11-13), and how a move is selected from the list of allowed moves for a particular neighborhood or move-type selected (line 8). Algorithm 2 outlines the neighborhood selection policy. The 
modified selectMove() procedure is given in Algorithm 3.

Local Minima Detection. A conformation $l$ is called a local minimum, if none of its neighbors has a lower fitness value, i.e. $f(l) \leq f(c)$ for all $c \in N$, where $N$ is the set of all neighbors $c$ of $l$. In our algorithm, we keep track of the trend of the fitness function and detect a local minimum by comparing its fitness function to that of the next selected candidate at the next iteration. Storing all possible local minimum is impractical, since it would then require huge time to search in the set of local minima. Hence we store only a proportion of the local minima encountered during the search. This selection is done randomly for the purpose of this paper and the effect of storing different proportions of local minima is described in the results section.

Representation. The solutions generated here are three dimensional Cartesian co-ordinates in the FCC lattice. They can be represented by the corresponding absolute direction between two points, which eliminates the problem of translational effects due to the moves performed. This type of representation is called the absolute encoding: every conformation $c$ is then represented by a string in the alphabet with 12 different symbols since the number of absolute directions or basis vectors in FCC lattice is 12 . However, the symmetry of the rotational effects is only eliminated by using the relative encoding. In this paper we followed the relative encoding scheme for FCC lattice proposed in [4] to represent a conformation. The alphabet size is also 12 when relative encodings are used.

Proximity measure. After a local minimum is detected, we store it in a list and at the time of candidate selection we search this list for similar conformations. We consider hamming distance for the similarity measure between two conformations. Given two conformations $c_{1}=$ "ABCAAADEF" and $c_{2}=$ "ABCGGGDEF", the hamming distance between $c_{1}$ and $c_{2}$ is $h\left(c_{1}, c_{2}\right)=3$, since they differ only in 3 positions. In our case, for any conformation $c$ and any local minimum $l \in L$ (where $L$ is the list of stored local minima), if the hamming distance $h(c, l) \leq r$ (where $r$ is the proximity measure), we say a re-visitation has occurred. For $r=0$, an exact stored local minimum is encountered. Whenever a re-visitation occurs, we discard the conformation and do not take the corresponding move. The hamming distance based similarity measure is correlated with the move used in this paper. Besides, it includes other conformations that have nearly matching representations within the proximity regions. Similar conformations have similar representations. Hamming distance thus can detect nearly similar conformations that are apparently distant based on move count between them. The effect of using different values for proximity measures is shown in the results section.

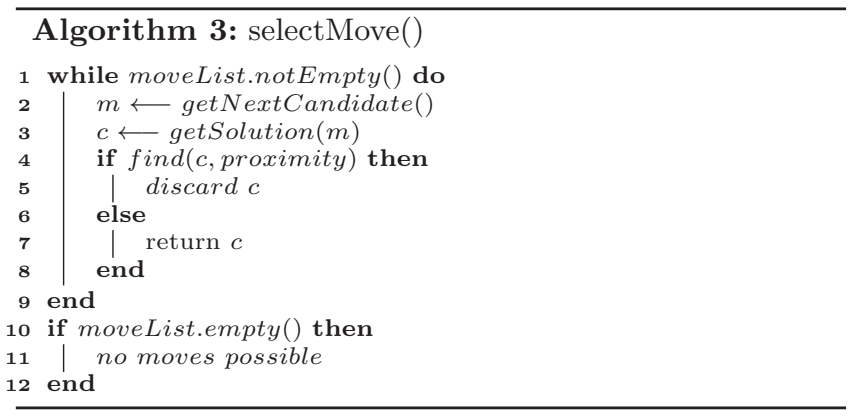

Selection of Moves. Algorithm 3 depicts how moves can be selected. At each step, we generate a solution from a selected move and then search the list $L$. If no similarity is found then we select it; otherwise we discard it. The get NextCandidate() method at line number 2 in Algorithm 3 returns the candidate move according to the move type selected in selecteNeighborhood() method.

Implementation. There are several issues regarding implementation. In this framework, the local search generates all possible moves based on the neighborhood and selects the one that minimizes the $\mathrm{H}-\mathrm{H}$ distances (in the case of $\mathrm{H}-$ moves) or randomly (in the case of P-moves). In our $\mathrm{C}++$ implementation, we maintain all the invariants needed to maintain the constraints and update the costs in an efficient way. It resulted in a very fast implementation which is almost 10 times faster than the COMET implementation [15]. However, our evaluations are mainly based on iterations.

Matching. The find(c,proximity) method called from the selectMove() procedure (line 4 in Algorithm 3) searches the list for a local minimum that is within the proximity measures of the selected candidate. The performance of this method depends on the data structure used in storing local minima and the matching technique of two conformations. In this paper, we implemented a simple array of compact byte-packed format to store the relative encoding of a conformation. It saved the memory space. Moreover, integer operations reduced the computational effort to find similarity. The matching is also faster due to the byte-packed format to store the conformations, since it allows integer operations to match. We take the bitwise xor of two packed conformations and search for equalities in parts of them. More efficient implementation of the memory is possible using complex data structures [6] and locality sensitive hashing techniques [34].

\section{EXPERIMENTS}

We compared the performance of our algorithm with the tabu search by Dotu et al. [15]. ${ }^{1}$ We ran experiments on the NICTA (www.nicta.com.au) cluster machine. The cluster has a number of machines, each equipped with two 6-core CPUs (AMD Opteron @2.8GHz, 3MB L2/6M L3 Cache) and 64GB Memory, running Rocks OS (a Linux variant for cluster). We ran both algorithms 50 times for each protein sequence. We were not able to compare our results with the hybrid method by Dotu et al. [15] that uses large neighborhood search, since we could not run the program on our system. The COMET program for the LNS approach exited with 'too much memory needed' error for the large size benchmark proteins that we have selected. We do not show results for small size Harvard instances (length $=48$ ) or other smaller proteins. The difference of the energy level achieved for these small proteins are relatively small, since both of the algorithms reach near optimal solutions.

\subsection{Results}

We produced results for two sets of benchmarks. We choose six large proteins that are also used by Dotu et al. [15]. Results for these six proteins are shown in Table 1. The $\mathrm{R}$ instances (length $=200$ ) are originally taken from [3] and the $\mathrm{f} 180$ instances (length $=200$ ) are provided by

${ }^{1}$ We thank Ivan Dotu for providing the COMET source code of their implementation. 
Table 1: Results for the R, f180 and instances taken from CASP

\begin{tabular}{|c|c|c|c|c|c|c|c|c|c|c|c|c|c|c|}
\hline \multirow{3}{*}{ Seq } & \multirow{3}{*}{ Len } & \multirow{3}{*}{$\mathrm{E}_{l}$} & \multirow{2}{*}{\multicolumn{4}{|c|}{$\begin{array}{c}\text { LS-Mem } \\
\text { titer }=1 \text { million }\end{array}$}} & \multicolumn{7}{|c|}{ LS-Tabu } & \multirow{3}{*}{ LNS } \\
\hline & & & & & & & \multicolumn{4}{|c|}{ \#iter $=1$ million } & \multicolumn{3}{|c|}{ timewise } & \\
\hline & & & best & mean & med & time & best & mean & med & time & best & mean & med & \\
\hline $\mathrm{R} 1$ & 200 & -384 & -353 & -325.11 & -326 & 0.66 & -333 & -318.14 & -318 & 2.28 & -324 & -310.54 & -311 & -330 \\
\hline R2 & 200 & -383 & -351 & -329.28 & -333 & 0.71 & -338 & -323.32 & -322 & 2.40 & $\mid-336$ & -315.98 & -316 & -333 \\
\hline R3 & 200 & -385 & -352 & -329 & -330 & 0.71 & 12 & -323.50 & -324 & 2.34 & -327 & -31 & -318 & 34 \\
\hline f180_1 & 180 & $?$ & -360 & -334.72 & -337 & 0.59 & |-500 & -325.26 & -325 & 2.21 & -333 & -319.5 & -319 & -293 \\
\hline f180_2 & 180 & ? & -362 & -340.26 & -343 & 0.65 & $\mid-348$ & -332.32 & -332 & 2.29 & 337 & -324.62 & -325 & -312 \\
\hline f180_3 & 180 & -378 & -357 & -343.12 & -343 & 0.63 & -350 & -337.62 & -340 & 2.26 & -346 & -33 & -333 & -313 \\
\hline no6 & 29 & -455 & -400 & -374.28 & -378 & 0.84 & $\mid-39$ & -369.86 & -371 & 2.83 & -376 & -35 & -360 & - \\
\hline $3 \mathrm{mr} 7$ & 189 & -355 & -311 & -291.74 & -292 & 0.64 & -301 & -287.06 & -288 & 2.52 & $\mid-292$ & -378.02 & -277 & - \\
\hline $3 \mathrm{mse}$ & 179 & -323 & -278 & -254.54 & -256 & 0.66 & -266 & -249.68 & -249 & 2.33 & -254 & -243.18 & -243 & - \\
\hline $3 \mathrm{mqz}$ & 215 & -474 & -415 & -386.7 & -385 & 0.62 & $\mid-401$ & -383.56 & -383 & 2.64 & -392 & -372.5 & -373 & - \\
\hline 3on7 & 279 & $?$ & -499 & -462.7 & -465 & 0.67 & -474 & -458.74 & -458 & 3.81 & -465 & -438.5 & -439 & 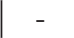 \\
\hline 3 no3 & 258 & -494 & -397 & -360.78 & -364 & 0.65 & -377 & -357.56 & -359 & 3.72 & $\mid-360$ & -342.38 & -343 & - \\
\hline
\end{tabular}

S.Will [15]. All these sequences can be found at the Peter Clote laboratory website. ${ }^{2}$ LS-Mem denotes our memory based local search and LS-Tabu denotes the tabu search by Dotu et al. [15]. We ran both algorithms for 1 million iterations using the same max Stable parameter set to 10000 as suggested in [15]. The best, mean, and median energy levels and the time are reported in Table 1 . To show the time wise performance comparison, we also present the best, mean, and median energy level achieved by LS-Tabu within the same time spent by our algorithm for each of the proteins. Note that the values in the time columns indicate time in hours. In the case of memory based approach, $10 \%$ of the local minima were stored and proximity measure set was set to 3 (for f180_3), 6 (for R1, f180_1 and f180_2 ) and 9 (for R2 and R3). Details about this parameter setting are explained later in this section. The best energy levels reported by Dotu et al. [15] are also shown under the column LNS. Optimal lower bounds of energy values for these proteins are also reported under the column ' $\mathrm{E}_{l}$ '. Note that these values are obtained by using exhaustive search methods [27]. ${ }^{3}$

We also derived a second set of benchmark from the target list of Critical Assessment of Techniques for Protein Structure Prediction (CASP) competition. ${ }^{4}$ We randomly chose six protein sequences of length $230 \pm 50$. We then converted them into HP sequences. Results for these six proteins are also given in Table 1. The PDB id for each of the proteins is also reported. The parameter proximity measure was set to 6 (for 3no6, 3mqz, 3on7), 9 (for 3no3) and 12 (for 3mse, $3 \mathrm{mr} 7)$. LNS column contains no data for these six proteins since they were not used in [15].

\subsection{Analysis}

From Table 1, it is evident that the memory-based approach finds conformations with significantly better energy levels when compared both iteration-wise and time-wise for all the 12 proteins. We performed statistical $t$-test for independent samples with $95 \%$ level of significance to verify the difference in performances. We report the new lowest energy

\footnotetext{
${ }^{2}$ http://bioinformatics.bc.edu/clotelab/FCCproteinStructure/ ${ }^{3}$ We thank Martin Mann for providing pre-calculated $\mathrm{H}$ cores needed to run CPSP tools.

${ }^{4}$ http://predictioncenter.org/casp9/targetlist.cgi
}

levels (which are shown in bold-faced font in Table 1) for all six proteins in the first set $(\mathrm{R}$ and $\mathrm{f180})$ w.r.t. incomplete search methods. We report the relative achievement of our approach measured in terms of the difference with the lower bound of energy level in Table 2 . This value is significant because it gets harder to find better conformations as the energy level of a protein sequence approaches the optimal. Relative Improvement $=\left(E_{o}-E_{r}\right) /\left(E_{l}-E_{r}\right) \times 100 \%$ where $E_{o}$ is the best energy level achieved by our approach, $E_{r}$ is the best energy level achieved by the tabu search, and $E_{l}$ is the lower bound of energy level. The values marked with * are those for which the CPSP tools didn't converge.

\begin{tabular}{|c|c|c||c|c|c|}
\hline \multicolumn{3}{|c||}{ Benchmark Set - I } & \multicolumn{3}{c|}{ Benchmark Set - II } \\
\hline Seq & $\mathrm{E}_{l}$ & Relative Imp. & Seq & $\mathrm{E}_{l}$ & Relative Imp. \\
\hline R1 & -384 & $39.21 \%$ & $3 \mathrm{no} 6$ & -455 & $15.38 \%$ \\
R2 & -383 & $28.88 \%$ & $3 \mathrm{mr} 7$ & -355 & $18.51 \%$ \\
R3 & -385 & $23.25 \%$ & $3 \mathrm{mse}$ & -323 & $10.52 \%$ \\
f180_1 & $-378^{*}$ & $55 \%^{*}$ & $3 \mathrm{mqz}$ & -474 & $19.17 \%$ \\
f180_2 & $-381^{*}$ & $42.4 \% *$ & 3on7 & - & - \\
f180_3 & -378 & $25 \%$ & 3no3 & -494 & $17.09 \%$ \\
\hline
\end{tabular}

Table 2: Relative Improvements of LS-Mem over LS-Tabu for the best energy levels achieved

\section{The Effect of Variation in Proximity Measure.}

In general, if we increase the proximity measure it results in an increased number of discarded solutions. The average hamming distance between two successive conformations is 3 due to the move used in this framework. We, therefore, ran experiments for different settings of proximity measures that are multiples of 3 . In the left graph in Figure 4, we show the effect of varying proximity measures for six different proteins. Other proteins show similar kind of behavior. For each of the cases, the number of discarded solutions increases as the proximity measure increases. For proximity measure set to 0 , the number of solution is in the range of hundreds and there is a sharp increase after proximity measure 3. The energy level achieved does not necessarily increase with the increment in the proximity measure. This is because after a certain level, for each protein, a high 

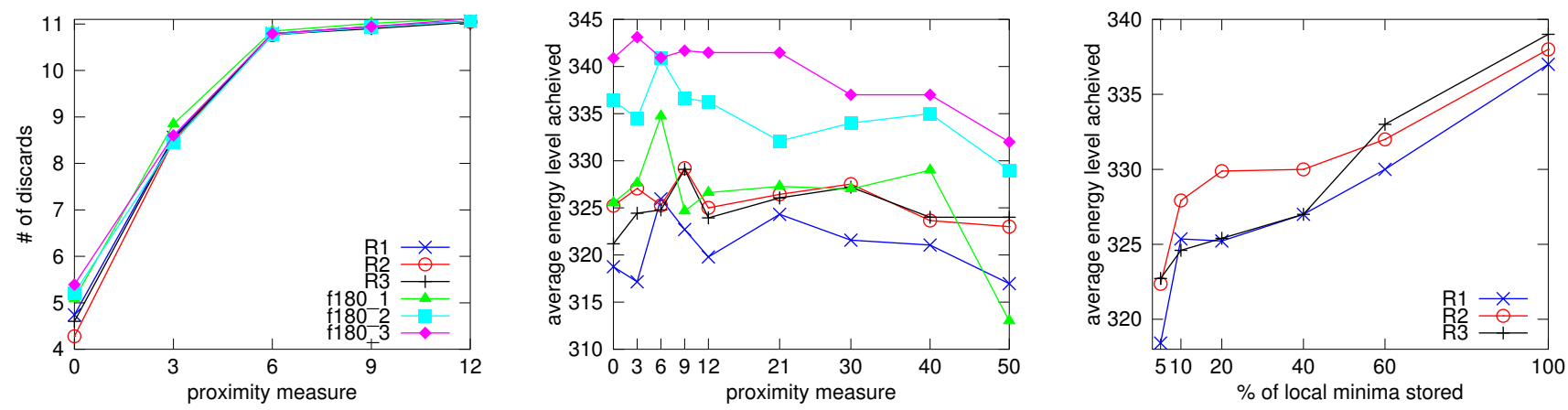

Figure 4: Plot of number of discards in log (left) and average energy level (middle) against proximity measures, energy levels achieved against percentage of local minima stored (right)

proximity measure causes discarding of a very large neighborhood, which may contain unvisited promising regions in the search space. The effect of variation in the energy level for different values of proximity measures are shown in the middle of Figure 4. These results were obtained by storing only $10 \%$ of the local minima.

\section{The Effect of Variation in Proportion of Stored Local} Minima.

The runtime performance degrades with the increase in the proportion, since it requires more time to search in a list with a larger number of local minima. For 1 million iterations with $5 \%$ of local minima stored, the runtime is usually around 20 minutes for the $\mathrm{R}$ sequences and it increases almost quadratically with an increase in the proportion. Moreover, the memory requirement increases linearly. For a protein with length $n$, the memory required for storing $k \%$ of the local minima is $O(k n) \mathrm{KB}$. However, the energy level generally shows a non-decreasing trend for different levels of proportion. For a fixed value of the proximity measure, increase in the proportion of local minima stored generally increases the search space covered and hence eliminates the scope of re-visitation; which results in better energy levels achieved. The effect is shown in the left graph in Figure 4.

\section{Duplicates generated within the search.}

With the same set of proteins shown in the table of Figure 2, our memory-based search was able to reduce duplicate generation for all the 4 proteins. The percentage of duplicates generated reduced from $40.92 \%$ to $16.7 \%$ for $\mathrm{H} 1,31.19 \%$ to $12.58 \%$ for P3, $42.53 \%$ to $3.28 \%$ for S1, and $39.34 \%$ to $6.46 \%$ for R1, where number of conformations stored were only 2003, 1253, 1128 and 761 respectively over 100,000 iterations.

\section{CONCLUSION AND FUTURE WORK}

In this paper, we have presented a memory-based local search for simplified protein structure prediction and shown the improvement of performance in comparison to state-ofthe-art methods on several standard benchmark proteins and report the lowest levels of energy for them. We have also shown the effects of choosing different proximity measure and storing different proportion of local minima. In future, we wish to explore the selection criteria for storing local minima. We think that the use of more efficient data structures like digital hash trees will improve the performance of the algorithm. We wish to test this memory-based search on similar problems and other variants of the protein structure prediction problem. In future, we wish to investigate the applicability of our approach in other local search methods such as genetic algorithms and constraint-based local search.

\section{REFERENCES}

[1] D. P. Alessandro, A. Dovier, and E. Pontelli. A constraint solver for discrete lattices, its parallelization, and application to protein structure prediction. Softw. Pract. Exper., 37:1405-1449, November 2007.

[2] C. B. Anfinsen. Principles that govern the folding of protein chains. 181(4096):223-230, 1973.

[3] R. Backofen and S. Will. A constraint-based approach to structure prediction for simplified protein models that outperforms other existing methods. Logic Programming, pages 49-71, 2003.

[4] R. Backofen, S. Will, and P. Clote. Algorithmic approach to quantifying the hydrophobic force contribution in protein folding. In Proceedings of the Pacific Symposium on Biocomputing, pages 92-103, 2000.

[5] D. Baker and A. Sali. Protein structure prediction and structural genomics. Science, 294:93-96, 2001.

[6] R. Battiti, G. Tecchiolli, et al. The reactive tabu search. ORSA journal on computing, 6:126-126, 1994.

[7] B. Berger and T. Leighton. Protein folding in the hydrophobic-hydrophilic(HP) is NP-complete. In Proceedings of the second annual international conference on Computational molecular biology, RECOMB '98, pages 30-39, 1998.

[8] J. Blazewicz, K. Dill, P. Lukasiak, and M. Milostan. A tabu search strategy for finding low energy structures of proteins in HP-model. Computational Methods in Science and Technology, 10(1):7-19, 2004.

[9] E. Bornberg-Bauer. Chain growth algorithms for HP-type lattice proteins. In Proceedings of the first annual international conference on Computational molecular biology, RECOMB '97, pages 47-55, New York, NY, USA, 1997. ACM. 
[10] M. Cebrián, I. Dotú, P. Van Hentenryck, and P. Clote. Protein structure prediction on the face centered cubic lattice by local search. In Proceedings of the $23 r d$ national conference on Artificial intelligence - Volume 1, AAAI'08, pages 241-246. AAAI Press, 2008.

[11] B. Cipra. Packing challenge mastered at last. Science, 281(5381):1267, 1998.

[12] P. Crescenzi, D. Goldman, C. Papadimitriou, A. Piccolboni, and M. Yannakakis. On the complexity of protein folding. Journal of Computational Biology, 5:597-603, 1998.

[13] S. E. Decatur. Protein folding in the generalized hydrophobic-polar model on the the triangular lattice. Technical report, 1996.

[14] I. Dotu, M. Cebrián, P. Van Hentenryck, and P. Clote. Protein structure prediction with large neighborhood constraint programming search. In Principles and Practice of Constraint Programming, pages 82-96. Springer, 2008.

[15] I. Dotu, M. Cebrian, P. Van Hentenryck, and P. Clote. On lattice protein structure prediction revisited. IEEE/ACM Trans. Comput. Biol. Bioinformatics, 8(6):1620-1632, November 2011.

[16] W. E. Hart and S. Istrail. Lattice and off-lattice side chain models of protein folding: Linear time structure prediction better than $86 \%$ of optimal (extended abstract). J. Comput. Biol, 4:241-259, 1997.

[17] W. S. Havens and B. N. Dilkina. A hybrid schema for systematic local search. In In Canadian Conference on Artifical Intelligence, 2004.

[18] T. Hoque, M. Chetty, and A. Sattar. Protein folding prediction in 3D FCC HP lattice model using genetic algorithm. In IEEE Congress on Evolutionary Computation, pages 4138-4145, 2007.

[19] T. Jiang, Q. Cui, G. Shi, and S. Ma. Protein folding simulations of the hydrophobic-hydrophilic model by combining tabu search with genetic algorithms. Journal of Chemical Physics, 119(8):4592-4596, 2003.

[20] N. Jussien and O. Lhomme. Local search with constraint propagation and conflict-based heuristics. Artif. Intell., 139(1):21-45, July 2002.

[21] H. Kawai, T. Kikuchi, and Y. Okamoto. A prediction of tertiary structures of peptide by the Monte Carlo simulated annealing method. 3(2):85-94, 1989.

[22] G. W. Klau, N. Lesh, J. Marks, and M. Mitzenmacher. Human-guided tabu search. In Proceedings of the 18th National Conference on Artificial Intelligence, pages 41-47, 2002.

[23] G. W. Klau, N. Lesh, J. Marks, and M. Mitzenmacher. Human-guided tabu search. In Proceedings of the 18th National Conference on Artificial Intelligence, pages 41-47, 2002.

[24] K. F. Lau and K. A. Dill. A lattice statistical mechanics model of the conformational and sequence spaces of proteins. Macromolecules, 22(10):3986-3997, 1989.

[25] N. Lesh, M. Mitzenmacher, and S. Whitesides. A complete and effective move set for simplified protein folding. In Proceedings of the seventh annual international conference on Research in computational molecular biology, RECOMB '03, pages 188-195, 2003.
[26] H. Lu and G. Yang. Extremal optimization for protein folding simulations on the lattice. Comput. Math. Appl., 57:1855-1861, June 2009.

[27] M. Mann, S. Will, and R. Backofen. CPSP-tools Exact and complete algorithms for high-throughput 3 D lattice protein studies. Bmc Bioinformatics, 9(1):230, 2008.

[28] B. Mazure, L. Sais, and É. Grégoire. Tabu search for sat. In Proceedings of the National Conference on Artificial Intelligence, pages 281-285, 1997.

[29] S. Miyazawa and R. L. Jernigan. Estimation of effective interresidue contact energies from protein crystal structures: quasi-chemical approximation. Macromolecules, 18(3):534-552, Mar. 1985.

[30] R. D. PalÃź, A. Dovier, and F. Fogolari. Constraint logic programming approach to protein structure prediction. BMC Bioinformatics, 5:2004, 2004.

[31] R. Samudrala, Y. Xia, E. Huang, and M. Levitt. Ab Initio protein structure prediction using a combined hierarchical approach. Proteins, 3:194-198, 1999.

[32] J. Schonbrun, W. Wedemeyer, and D. Baker. Protein structure prediction in 2002. Current opinion in structural biology, 12(3):348-354, 2002.

[33] A. Shmygelska and H. Hoos. An ant colony optimisation algorithm for the $2 \mathrm{D}$ and $3 \mathrm{D}$ hydrophobic polar protein folding problem. $B M C$ bioinformatics, 6(1):30, 2005.

[34] M. Slaney and M. Casey. Locality-sensitive hashing for finding nearest neighbors [lecture notes]. Signal Processing Magazine, IEEE, 25(2):128-131, March 2008.

[35] A. D. Ullah, L. Kapsokalivas, M. Mann, and K. Steinhöfel. Protein Folding Simulation by Two-Stage Optimization. In Z. Cai, Z. Li, Z. Kang, \& Y. Liu, editor, Computational Intelligence and Intelligent Systems, page 138, 2009.

[36] A. Z. M. D. Ullah and K. Steinhöfel. A hybrid approach to protein folding problem integrating constraint programming with local search. $B M C$ Bioinformatics, 11(S-1):39, 2010.

[37] R. Unger and J. Moult. A genetic algorithm for three dimensional protein folding simulations. In Proceedings of the 5th International Conference on Genetic Algorithms, pages 581-588, 1993.

[38] P. Van Hentenryck and L. Michel. Constraint-based local search. The MIT Press, 2009.

[39] S. Will. Exact, constraint-based protein structure prediction in simple models. PhD thesis, Friedrich-Schiller-University Jena, 2005.

[40] K. Yue and K. Dill. Forces of tertiary structural organization in globular proteins. Proceedings of the National Academy of Sciences of the United States of America, 92(1):146, 1995.

[41] K. Yue, K. Fiebig, P. Thomas, H. Chan, E. Shakhnovich, and K. Dill. A test of lattice protein folding algorithms. Proceedings of the National Academy of Sciences, 92(1):325, 1995. 